\title{
Quantum Bubble Polarization in Curved Spacetime
}

\author{
Alexander Yosifov \\ Shumen, Bulgaria \\ Email: alexander_yosifov@abv.bg \\ Received 4 March 2016; accepted 15 April 2016; published 18 April 2016 \\ Copyright (C) 2016 by authors and Scientific Research Publishing Inc. \\ This work is licensed under the Creative Commons Attribution International License (CC BY). \\ http://creativecommons.org/licenses/by/4.0/ \\ (c) (i) Open Access
}

\begin{abstract}
The conventional singular hot big bang scenario is questioned. A new model which does not include an initial singularity $g_{00}=\infty$ at $t=0$, neither a brief period of exponential expansion $a(t) \sim \mathrm{e}^{H t}$ is considered. The main parameters $T$ and $\rho$ are kept finite. The beginning of the universe we occupy is revisited in the framework of quantum field theory in curved spacetime. However, a straightforward alternative mechanism for not only solving the most fundamental problems in modern cosmology-flatness problem, horizon problem and magnetic monopole problem, but even suppressing their number is provided. In the particular paper, we discuss the essential role quantum entanglement plays in the structure of the spacetime and the apparent contradiction between quantum mechanics and general relativity in terms of classical field theory in $3+1$ dimensions.
\end{abstract}

\section{Keywords}

\section{Cosmology, Big Bang, String Theory}

\section{Introduction}

For the past decades, the prevailing view, regarding the beginning of the universe and its evolution in the first fraction of a second, has been a combination of the big bang theory and the theory of cosmic inflation [1]. However, recently this paradigm has been challenged. According to the big bang theory, if we extrapolate the current picture of the universe backwards in time, temperature $T$ and density $\rho$ start increasing until we reach the initial singularity $g_{00}=\infty$ at the beginning of time $t=0$. At that moment, the whole universe is compressed to a single point of zero size, $t \rightarrow 0$ and $a(t) \rightarrow 0$ while $T \rightarrow \infty$ and $\rho \rightarrow \infty$. At this point, our laws of physics break down. The central problem of the big bang theory is namely the singularity. Furthermore, the theory does not provide any explanation regarding the initial conditions. The model is completely ignorant about the events prior to the expansion phase as neither space, nor time existed. The theory of cosmic inflation states that right after the big bang 
the universe goes through a brief period, $10^{-36} \mathrm{~s}$., of superluminal exponential expansion $a(t) \sim \mathrm{e}^{H t}$. The concept is originally pioneered to smooth and flatten the universe, starting from random initial conditions, and thus solve the horizon, flatness and magnetic monopole problems with a straightforward mechanism. The conventional theory dictates that in order for inflation to be triggered, a scalar field $\varphi$, inflaton, satisfying the property $V(\varphi) \gg E_{K}$ has to be presented. Because of the small kinetic energy $E_{K}$, the inflaton $\varphi$ settles down to a state of potential well adiabatically. Once the scalar field $\varphi$ is settled, the potential energy $V(\varphi)$ starts dominating. When the potential takes over, the Lagrangian $L=E_{K}-V(\varphi)$, becomes negative and the universe starts expanding. The measure of the expansion, e-folds, is given by $\left(a\left(t_{f}\right)\right) /\left(a\left(t_{i}\right)\right)=\mathrm{e}^{N}$, where $a\left(t_{f}\right)$ and $a\left(t_{i}\right)$ are the scale factor after and prior to inflation, respectively. The number of e-folds, $N$, is defined as $N \equiv\left(t_{f}-t_{i}\right) H$, where $H=\dot{a} / a$, is the Hubble parameter. It should be noted that large kinetic energy value will prevent inflation from initiating. We are not going to provide a detailed description of inflation, neither will we examine its different models, as this is not of interest to the particular paper. Great effort in this direction has been devoted in the past [2]-[4]. However, this view has to be abandoned. Recent data by the Planck satellite, [5], challenges the contemporary paradigm. A subsequent paper, based on the obtained results, [6], shows problems with inflation that until now are not present. According to the data, for inflation to start smoothing and flattening the universe, the universe has to have extremely low initial anisotropy prior to the exponential expansion phase. Moreover, Planck 2013 rules out most of the inflationary models and favors only the simple ones [7]-[9]. It shows we live in an amazingly elegant universe; the spatial curvature is negligible and the fluctuations are Gaussian. The overall data calls for a new and simple description of the universe. The contemporary cosmological model suffers from many problems which force us to rethink our understanding concerning the early history of the universe. We discuss an alternative non-singular and inflation-free model which predicts finite values for both space and time. The proposed picture is simple in a sense that it does not require the addition of $n$ compactified extra dimensions. The model eliminates the flatness of the spacetime geometry as a problem, and solves both of the remaining ones, horizon and monopoles problems, with a straightforward mechanism motivated by certain string theory models.

The paper is organized as follows. In Sec. II we present a model for the beginning of the universe which does not include rapid entropy production and describe its main features. Using arguments from the no-hair theorem and the holographic principle we claim there are similarities between the boundary surface of a bubble universe and the holographic representation of a black hole event horizon. Later in the section we develop a mechanism for creating a new universe using quantum field theory in curved spacetime (QFTCS) starting from basic assumptions. In Sec. III we address the fundamental cosmological problems without considering inflation and provide alternative solutions. Further, in Section IV, we examine the role quantum entanglement has in the early universe, and how certain high-energy corrections to it might explain the structure of the spacetime, thus addressing the present issues of the origin of the cosmological principle and the spooky action at a distance. Moreover, we put forward a novel way for explaining the cosmological principle by making certain assumptions regarding the structure of spacetime in terms of tiny wormholes, and provide a mechanism for their natural arising.

\section{Bubble Multiverse}

We will describe a scenario which provides a non-inflationary solution to the horizon and magnetic monopoles problems. The model represents each universe as a separate 3+1 dimensional bubble, described by the Friedmann-Robertson-Walker (FRW) metric

$$
\mathrm{d} s^{2}=-\mathrm{d} t^{2}+a^{2}(t)\left[\frac{\mathrm{d} r^{2}}{1-k r^{2}}+r^{2} \mathrm{~d} \Omega^{2}\right] \equiv g_{\mu \nu} \mathrm{d} x^{\mu} \mathrm{d} x^{v}
$$

where $\mathrm{d} \Omega^{2}=\mathrm{d} \Theta^{2}+\sin ^{2} \Theta \mathrm{d} \varphi^{2}, k=+1$ in which case the scale factor $a(t)$ becomes the radius curvature of space, denoted as $R(t)$. Einstein's equation for the particular metric reads

$$
G_{\mu}^{v}=\frac{8 \pi G}{3} T_{\mu}^{v}
$$

where $T_{\mu}^{v}$ is the energy-momentum tensor. The Friedmann equation for the evolution of the FRW universe is 


$$
\left(\frac{\dot{a}}{a}\right)^{2}=(H(t))^{2}=\frac{8 \pi G}{3 c^{2}} \varepsilon(t)-\frac{k c^{2}}{R_{0}^{2} a(t)}
$$

where $k=+1, \varepsilon(t)$ is the energy density and $a(t)$ is the FRW scale factor. Initial homogeneity and isotropy of the universe on large scales (>100 Mpc) is assumed. The bubbles are taken to propagate in a quantum vacuum background and interact only via gravity. Hence, we suppose different bubbles can approach each other. This is a semi-classical theory, matter fields are quantized and gravity is treated classically. In the presence of a gravitational source the spacetime geometry becomes non-Euclidean, thus allowing for trivial description of the dynamics using Einstein's field equations

$$
G_{\mu \nu}=8 \pi\left\langle T_{\mu \nu}\right\rangle
$$

Given the metric of the spacetime, depending on the matter and radiation density, one might expect the universe to reach a maximum size $R_{\max }$ at $\dot{R}=0$, where $\dot{R}=\mathrm{d} R / \mathrm{d} t$, and then start contracting or keep on expanding monotonically and reaching infinite size in the asymptotic future $a(t) \rightarrow \infty$ as $t \rightarrow \infty$. In the framework we describe, it is more convenient to assume finite size of the different universes. It is therefore plausible, we believe, to assume every single bubble has a boundary layer mimicking superfluid properties. We are particularly interested in the forces of repulsion which appear between them. As a result, when two "parent" universes come close together, due to the superfluid properties of their shells, a force of repulsion, that overcomes the force of gravity, occurs. Hence, the bubbles repel each other. Because of the strong gravitational field, generated by the two universes, the quantum vacuum in-between gets polarized. The idea of vacuum polarization in the presence of a strong gravitational field was presented by Hawking [10]-[14]. He proved mathematically that at the vicinity of its horizon, $g_{00}=0$, a black hole polarizes the quantum vacuum under the influence of its own gravitational field, which leads to an increase of the local energy density of the quantum fluctuations $\delta \varphi$, hence pairs of positive frequency oscillations are produced. The expectation value of the field fluctuations in curved spacetime is given by $\left\langle\varphi^{2}\right\rangle$. The change of the area of the event horizon due to the particle absorption, is non-negative. Consequently, high frequency outgoing modes of the quantum field, Hawking radiation, are emitted to infinity and the black hole evaporates. A static black hole emits Hawking quanta with a black body thermal spectrum of temperature $T=\frac{\hbar c^{3}}{8 \pi G M}$, where $\hbar$ is the reduced Planck constant. Because of the quantum nature of the environment (bulk), it is convenient to use quantum field theory in curved spacetime (QFTCS) when describing the mechanism for producing a new universe. The no-hair theorem [15] [16] implies black holes are indistinguishable from one another. It posits they are entirely described by three classical parameters-angular momentum $J$, charge $Q$, and mass $M$. The theorem states all of the information regarding the matter that has collapsed to form the black hole is trapped behind the event horizon and is inaccessible to external observers. Similarly, bubble universes, as viewed by an outside observer, can be described by the same parameters. This line of reasoning allows us to assume the boundary surface of an individual bubble is a flat plane provided the bubble's radius is big enough. This was first proposed by 'tHooft in the context of applying the holographic principle to regions with large volume. We have arrived to the same conclusion based on completely different arguments which suggests the proposed view might have deep implications. The similarities found between a boundary layer of a universe and a black hole event horizon allow us to treat them analogously. Describing the quantum vacuum polarization in the spacetime region between two bubble universes, we apply the Hermitian operators $\Psi^{\dagger}(x)$ and $\Psi(x)$ to the scalar field $\varphi$. They are defined as follows

$$
\Psi^{\dagger}(x)=\sum_{i} \psi_{i}^{*}(x) a_{i}^{?}, \Psi(x)=\sum_{i} \psi_{i}(x) a_{i}
$$

where $a_{i}^{\dagger}$ and $a_{i}$ are the creation and annihilation operators, respectively. When we apply a creation operator on the lowest possible energy state, the vacuum $|0\rangle$, we get

$$
a_{i}^{\dagger}|0\rangle=|x\rangle
$$

We find that the creation operator produces a one-particle quantum state from the vacuum at point $x$. This is due to the instability of the quantum vacuum in the presence of a strong gravitational field. The initial vacuum will not appear entirely particle-free to all observers. An annihilation operator, acting on the vacuum yields 


$$
a_{i}|0\rangle=|0\rangle
$$

for all states.

The number of particles in this case is not globally determined but rather observer-dependent. An integral part of matter field polarization (particle production) is the observer-dependence of the definition of a particle. Let us consider the following example. Suppose we have two observers, Alice and Bob, who move along their respective worldlines. As they do they carry a measuring apparatus to count the number of particles they encounter, $N_{i}$. What we find is that the number of particles which Alice and Bob measure is different, thus $N_{A} \neq N_{B}$. Defining positive frequency oscillations in curved spacetime is non-trivial. Consider the following. Suppose, for the sake of simplicity, that as two vacuum bubbles are close together, we turn gravity off. Since the gravitational field is off, there will be no particles produced from the vacuum $|0\rangle$. If we now turn the field back on again, we will measure a production of certain number of particles due to the time-dependence of the gravitational field. The expectation value of the particle production is given by

$$
\left\langle N_{i}\right\rangle=\left\langle 0\left|a_{i}^{\dagger} a_{i}\right| 0\right\rangle
$$

where $N_{i}$ is the number operator and it is defined as $N_{i}=a_{i}^{\dagger} a_{i}$. If the expectation value $\langle N\rangle$, is non-zero then there are particles produced by the gravity field. The above developed concept is essential to the mechanism for producing new vacuum bubbles. Let us provide another example for better justifying the notion of observer-dependence of the particle productuion. Quantum field theory is assumed to be gauge invariant. Thus gauge transformations should not result in any change of the field $\varphi$. However, when we use classical perturbation theory we get non-gauge invariant results. Particle numbers, as we have already argued, are defined in terms of creation and annihilation operators, and differ depending on the frame of reference. Suppose we decompose the field $\varphi$ into creation and annihilation operators denoted as $a_{i}^{\dagger}$ and $a_{i}$, respectively. Following the previous example, let us describe the physics in terms of different frames of reference. We decompose the field $\varphi$ as follows

$$
\begin{gathered}
\varphi=\sum_{i}\left(a_{i} f_{i}+a_{i}^{\dagger} f_{i}^{*}\right) \\
\varphi=\sum_{i}\left(b_{i} f_{i}+b_{i}^{\dagger} f_{i}^{*}\right)
\end{gathered}
$$

Again, we find that different observers measure different values for the number operator $N$, hence $a_{i} \neq b_{i}$. Thus if we have a Hilbert space $\mathcal{H}$, in a certain state $|\psi\rangle$, the state will be described differently by the different observers. This is an effect which arises as gravity acts on the quantum field $\varphi$. We expand the creation and annihilation operators by a Bogoliubov transformation

$$
a_{i}=\sum_{j}\left(\alpha_{i j}^{*} a_{j}-\beta_{i j}^{*} a_{j}^{\dagger}\right)
$$

where $\alpha_{i j}$ and $\beta_{i j}$ are the Bogoliubov coefficients. The scalar fields are time-independent; they solely depend on position. Therefore we assign a Hermitian operator to every point in space

$$
\Psi^{\dagger}(x)=\sum_{i} \Psi_{i}^{*}(x) a_{i}^{\dagger}
$$

We can calculate the density by applying the operators $\Psi^{\dagger}(x)$ and $\Psi(x)$. Integrating over a particular region allows us to find the approximate number of particles in the given volume of space

$$
\begin{gathered}
\int \mathrm{d} x \Psi^{\dagger}(x) \Psi(x) \\
\int \mathrm{d} x \sum_{i j} a_{i}^{\dagger} \Psi_{i}^{*}(x) a_{j} \Psi_{j}(x) \\
\sum_{i j} a_{i}^{\dagger} a_{j} \delta_{i j}
\end{gathered}
$$

Due to the quantum nature of the process the number varies. The Dirac delta function shows continues distribution. We can now proceed and calculate the energy density in the present region. We will start by first considering the more familiar example with only one particle. The energy of each produced quanta is given by the time-independent one-particle Schrodinger equation 


$$
\Psi_{i} H=\Psi_{i} \omega
$$

where $H$ is the Hamiltonian. Substituting $H$ in Equation (12) gives us

$$
\Psi_{i}\left[\left(\frac{-\hbar^{2}}{2 m}\right) \nabla^{2}+V(\varphi)\right]=\Psi_{i} \omega_{i}
$$

where $\nabla^{2}$ is the Laplace operator and in 3D Cartesian coordinates yields the form $\nabla^{2}=\frac{\partial^{2}}{\partial x^{2}}+\frac{\partial^{2}}{\partial y^{2}}+\frac{\partial^{2}}{\partial z^{2}}$.

Hence, by applying Hermitian operators to the Hamiltonian of each one-particle state we find the total amount of energy in a given region of space to be

$$
E=\int \mathrm{d} x \Psi^{\dagger}(x)\left[\left(\frac{-\hbar^{2}}{2 m}\right) \nabla^{2}+V(\varphi)\right] \Psi(x)
$$

Let us now consider the many-particles case. Despite we are now dealing with a more complex system, consisting of $N$ number of particles, and one might expect it to be more complicated, we do, however, apply the same formalism as in Equation (13)

$$
H=-\frac{\hbar^{2}}{2} \sum_{i}^{N} \frac{\nabla_{i}^{2}}{m_{i}}+V(\varphi)
$$

where the dot product of the del operator denotes the kinetic energy of the $N$-particle system. The above developed concept creates the initial causally connected patch of matter which will later expand to become our universe. We initially begin with a non-zero value of the scale factor $a(t)$, thus we avoid the formation of a cosmic singularity and keep the parameters $\rho$ and $T$ finite. The matter patch is in low entropy state and must satisfy the extremely low initial anisotropy condition in order for expansion to be initiated. Considering an inflation-free model, depending chiefly on the initial expansion rate $\Lambda$, leads to two extreme possible scenarios in the early universe: (i) adiabatic expansion and (ii) very rapid initial expansion, corresponding to $\Lambda \ll 1$ and $\Lambda \gg 1$, respectively. In the first case, the critical value of the density $\rho_{\text {crit }}$ will be exceeded quickly, thus gravitational attraction will take over, resulting in an immediate collapse. Whereas in the second case, numerous Planckian-size low entropy state horizons will form, each of which containing several bits of information. In both cases the lifespan of the universe will be incredibly short; thus being, for all practical purposes, meaningless. Aside from the extreme scenarios, for the universe to grow and form large-scale structures, the initial expansion $\Lambda$, has to take an arbitrary value somewhere between the extreme velocities

$$
\Lambda \ll 1<\Lambda_{\text {right }}<\Lambda \gg 1
$$

\section{Cosmological Problems}

A period of chaotic exponential expansion is not required in order for the main cosmological problems to be solved. Alternative solutions are presented in the present section. We argue the apparent flatness of the spacetime geometry, at least on the scales we observe, should not be considered a problem but rather a consequence. The metric of the universe depends chiefly on two parameters, $\rho$ and $H$. A stable closed model, however, can be constructed. A non-singular patch with extremely low degree of initial anisotropy in low entropy state, going through a non-exponential expansion phase, can result in an apparent flatness. Achieving spacetime flatness in models which do not include exponential entropy production in the early universe is essential part of cyclic cosmology. The contemporary cyclic scenarios rely on ekpyrotic phase with equation of state $w \gg 1$ to occur as the universe monotonically contracts towards a crunch/bang transition point in order to set the conditions straight. During the slow-contraction period prior to the rebounce (big bang) nearly scale-invariant perturbations are produced. As the universe contracts certain energy components start to dominate, thus decreasing the anisotropies and the curvature. Therefore, each cycle begins in a high-isotropy low-entropy state. As we have mentioned earlier in the paper, the primordial non-singular patch needs to be in low entropy state and have extremely low initial anisotropy in order to start expanding. These requirements follow from the Planck 2013 data and strongly oppose the chaotic inflationary scenarios. We find vital similarities between the conditions set during the ekpyrotic phase in cyclic cosmology and the initial conditions required for expansion to start on first place. Therefore, the low entropy and the 
extremely low degrees of initial anisotropy, which the primordial patch must possess, are generic (natural) requirements. To achieve flatness of the spacetime metric one needs to have almost perfect unity of $\Omega$, thus $\Omega=\frac{\rho}{\rho_{\text {crit }}}$, where $\rho$ is the current density and $\rho_{\text {crit }}=\frac{3 H^{2}}{8 \pi G}$. Detailed calculations are provided in [17] and [18]. The $4 \mathrm{~d}$ spacetime within the bubble universe we live in might appear flat on smaller scales. Although one can set local coordinates that exhibit Minkowski metric, they do not represent the complete manifold. It is possible, however, for the universe to exhibit flat geometry on fairly large scales $\left(>\mathrm{H}^{-1}\right)$. Based on the requirements for expansion to be initiated, the flatness of the spacetime, observed today, is simply a natural consequence of the initial conditions.

Let us now consider the horizon problem. A non-inflationary solution which was inspired by some string theory models is proposed. Suppose the existence of a tachyon field in the low entropy state of the universe. The tachyon field coupled to $4 \mathrm{~d}$ gravity has a non-canonical action which can be written as

$$
S_{T}=-\int \mathrm{d}^{4} x \sqrt{(-g)}\left[V(T) \sqrt{\left(1+\partial_{\mu} T \partial^{\mu} T\right)}\right]
$$

where $V(T)$ is the positive effective potential of the field with maximum value at $\varphi=0$ and $g$ is the coupling constant. The effective potential is given by

$$
V(T)=\frac{\lambda}{4}\left(\varphi^{2}-v^{2}\right)^{2}
$$

The Lagrangian of the scalar field in curved spacetime is written as

$$
L(x)=\frac{1}{2}[-g(x)]^{1 / 2}\left[g^{\mu v}(x) \partial_{\mu} \varphi(x) \partial_{\nu} \varphi(x)-\left[m^{2}+\varepsilon R(x)\right] \varphi^{2}(x)\right]
$$

where $m$ is the mass of the field, $R$ is the Ricci scalar and $\varepsilon$ is the coupling constant. The tachyon field is in a state of unstable equilibrium at the top of its potential energy $V(\varphi)$. Due to quantum fluctuations $\delta \varphi(x)$, the field $\varphi$ is taken out of its present state, consequently, it starts oscillating, thus producing metastable spin- 0 electrically neutral particles (tachyon condensate) by converting all of its effective potential. It takes only a single oscillation because the quantum fluctuations $\delta \varphi(x)$, grow exponentially and quickly begin to dominate the dynamics, thus the field $\varphi$ very rapidly fills the universe homogeneously. The produced quanta, tachyons, obey the following energymomentum relation $E^{2}=p^{2}+m^{2}$, where $c=1$. Because the tachyon particles are metastable, they dilute before their energy reaches zero, as this would imply infinite propagation velocity, $v_{T} \rightarrow \infty$ as $E \rightarrow 0$. As the field rolls down, it starts oscillating and relaxes to a stable configuration (potential well), corresponding to the minimum of its $V(\varphi)$. Once the field is settled, no more particles are produced. The tachyons propagate superluminally for an extremely brief period of time. When the tachyons dilute, they release the remaining of their energy. Therefore they can even the temperature of the universe and produce the near-isotropy of the cosmic microwave background (CMB) radiation.

The apparent lack of monopoles in the universe today is one of the central puzzles in modern cosmology. We do, however, strongly believe in their existence. Contemporary particle models, like Grand Unified Theories (GUTs) and superstring theory predict the existence of magnetic monopoles. Furthermore, experiments for artificially creating monopoles bolster our view even more [19]. Most of the Grand Unified Theories suggest that when the temperature of the early universe dropped below the GUT threshold $T<T_{G U T}$, the universe went through a phase transition, associated with spontaneous symmetry breaking, and hence the creation of topological defects, like domain walls, cosmic strings, and magnetic monopoles, for example. The rest energy of the magnetic monopoles at the time of the GUT phase transition, based on most particle models, is estimated to be $m_{M} c^{2}=10^{12} \mathrm{TeV}$, which yields an approximate energy density of $\rho_{M} \sim 10^{94} \mathrm{TeV} \cdot \mathrm{m}^{-3}$. We argue the provided above string theory mechanism, tachyon instability, solves two of the fundamental cosmological problems - horizon and monopoles problems, simultaneously. As already argued, we assume the existence of a quantum field, tachyon field, which due to quantum fluctuations $\delta \varphi(x)$, is taken out of its unstable equilibrium state, and hence produces metastable tachyon particles. When we address the monopoles problem we focus on a specific feature of the tachyon particles. Namely, the inversed proportionality between their energy $E_{T}$ and velocity $v_{T}$. Thus $v_{T}$ exponentially increases as $E_{T}$ exponentially decreases, following the straightforward relation: $E_{T}=1 / v_{T}$. The released tachyon condensate 
leads to very rapid decrease of the density distribution of monopoles in the early universe. The simple energy/velocity relation places an upper mass limit to the monopole particles of approximately $10^{15} \mathrm{GeV}$. The exponential decrease of the energy of the produced condensate forces the tachyons to quickly fall below the treshold for annihilating monopoles. Therefore, we expect only heavier versions of the monopoles $\left(10^{15} \mathrm{GeV}\right)$ to be abundant. Because of the extremely short time scale $\left(\sim t_{p}\right)$ tachyons do not have enough time (thus energy) to annihilate the heavy monopole particles. The placed upper mass limit is of great importance. Given the high density of the monopoles in the early universe $\left(\sim 10^{94} \mathrm{TeV} \cdot \mathrm{m}^{-3}\right)$, higher mass limit would have caused gravitational instability, and hence collapse.

\section{Conclusions and Discussion}

In the present section, we will first discuss the apparent contradiction between quantum mechanics and general relativity by conducting a simple gedanken experiment. We will then present an early-universe phenomena which reinterprets the nature of the spacetime by introducing a network of tiny wormholes or maxiamlly correlated general states. The scenario occurs naturally as we approach Planck energy scale and address the cosmological principle which we have been taken for granted. The phenomena show the crucial role quantum mechanics plays in the early history of the universe. A lot of work towards unifying quantum mechanics and relativity has been done in the past. However, developing a comprehensive theory of quantum gravity has proven to be extremely difficult. The conclusions, which we draw, are based on a gedanken experiment which we will now put forward. For the purposes of the current experiment, we will consider the fundamental building blocks of nature to be tiny constructor pieces which behave quantum mechanically. Imagine Charlie starts playing with the constructor pieces by adding the individual blocks together. We assume he has created a bigger structure. Although the new construction is somewhat bigger, suppose it still behaves probabilistically. If he keeps playing though, a point will be reached, at which the whole system (consisting of $N$ number of pieces) will start exhibiting deterministic properties. However, consider Charlie decides to take a piece away from his toy, so that the new structure now consists of $N-1$ pieces. As a result, we assume that his actions will bring back the random nature of the system. The difference in the behavior between the $N$ number configuration and the $N-1$ number configuration is believed to be discrete. Following the gedanken experiment described above, we narrow it down to two possibilities. The first possibility relies on the well-bounded difference between the intuitive laws in the macroscopic world and the seemingly chaotic laws in the quantum realm. That is why it is plausible to assume that at particular scale a transition between quantum mechanics and general relativity occurs. Building on that, it is then feasible for us to speculate that every $N$-particle quantum system will change its nature to general relativistic one in the $N+$ 1-particle case when the transition point, corresponding to a certain number of particles, is passed. However, it is still unknown at what scale exactly does the transition between stochastic and deterministic behavior occur. The second interpretation of the gedanken experiment includes the naive conclusion that since the fundamental building blocks of nature act quantum mechanically, then the large-scale structures they make should act in the same manner. In this case, the reason why we tend to experience our macro world as deterministic can be attributed to our ability to perceive the physical reality. It should be noted that we have covered the second possibility despite the skepticism towards it. Based on the provided interpretations of the thought experiment, we argue that general relativity is nothing more than a macroscopic manifestation of quantum mechanics. A contradiction is present because we are trying to unify a manifestation of a theory with the theory itself. An interesting connection to a paper by 'tHooft [20] is present. 'tHooft makes the arguement that holographic description of nature only makes the model more far fetched to reality. However, spacetime appears to be fundamentally non-local because of its combinatorial structure held together by quantum entanglement. As we have argued in the current work, everyday physics obeys locality because it is simply a low-energy approximation. This being said, models which feature holography should not be considered as being far fetched. We might even get a step further and argue that since at super-Planckian regimes $\left(>M_{P}\right)$ there is no notion of locality, there should not be any notion of time either. This, of course, being highly speculative argument. Hence locality and time are emergent low-energy effects. Because we have argued that quantum mechanics acts differently in high-energy regimes, one, in fact, might make the case that quantum mechanics is indeed a part of a deeper framework. However, a more conservative approach to the argument would be that the difference is not a harbinger that it is part of a deeper theory but rather that at such energies certain high-energy corrections have to be made.

As we have already explicitly stated in the paper above, quantum field theory in curved spacetime provides the 
mechanism for solving the singularity problem $\left(g_{0}=\infty\right)$. The technique developed in Section II is what gives rise to the non-singular patch of matter which, if it satisfies certain initial conditions, expands to become our universe. Thus at no point did we talk about geodesically incomplete spacetime regions or infinities regarding $T$ and $\rho$. We start talking about entanglement only after the primordial patch has been created, and hence the singularity problem has been solved. The energies present at that point, we believe, may exceed the $M_{p}$, and thus give natural rise to the notion of "global entanglement". Therefore, the patch of matter is maximally entangled and this could have placed the seeds for the low-energy approximation monogamy of entanglement we observe today.

The homogeneity and isotropy of the universe on large scales $(>100 \mathrm{Mpc})$ is formulated as the cosmological principle. A satisfactory explanation about which has not yet been provided, and the very notion has been so far taken for granted. As we go backwards in time both the temperature and the density of the universe increase. If we go as far back as $10^{-36} \mathrm{~s}$. The temperature of the universe is approximately $10^{28} \mathrm{~K}$, corresponding to energy levels of $10^{12} \mathrm{TeV}$. At that energy scale the electromagnetic, weak and strong forces unify in Grand Unified Theory (GUT). We expect if we go even further back in time to $10^{-43} \mathrm{~s}$. the temperature of the universe to be $10^{33} \mathrm{~K}$, corresponding to Planck energy, $E_{p} \sim 10^{16} \mathrm{TeV}$. This is believed to be the so-called Theory of Everything scale, at which gravity unifies with the other gauge forces. I claim quantum mechanics acts differently in a certain way when $T \gg T_{G U T}$. Therefore, we argue the monogamy of entanglement is violated at Planck energy scale and the so-called "global entanglement" is present. Let us clarify our understanding of "global entanglement". As it is already well-known, monogamy of entanglement states that if two qubits are maximally correlated, they cannot be correlated with third party (qubit). Hence

$$
\left|\Phi_{A B}\right\rangle=\frac{1}{\sqrt{2}}(|00\rangle+|11\rangle)
$$

As we have already stated, we believe it is possible to assume that in high-energy regimes when $T \geq M_{P}$, quantum mechanics acts differently and the monogamy of entanglement is violated. Thus the above correlation Equation (19) is extended to $n$ number of parties.

The notion of global entanglement in the very early universe can naturally explain the cosmological principle we observe today. In that sense, objects separated by distance greater than the Hubble radii never lose causal contact, as one might expect. On a deeper quantum mechanical level the different regions of the universe are always in causal contact, regardless of the physical distance. Because of the fundamental implications of quantum mechanics, it is plausible for us to assume the global entanglement played an essential role in the early history of the universe. Recent developments in theoretical physics opened the possibility for a different approach to the nature of the spacetime. Work by Mark Raamsdonk [21] and others point out that we might have to reconsider our understanding of the spacetime. A proposal was made that the classical spacetime geometry emerges from quantum entanglement. In a sense, quantum entanglement holds space together and the structure of the spacetime is combinatorial rather than continues. The contemporary, and somewhat radical views, fit elegantly with the idea of polygamy of entanglement at Planck energy scale. In this picture the "spooky action at a distance" becomes a feature of the combinatorial structure of the universe. The naive interpretation would be that in a combinatorial structure, held together by quantum entanglement, any two points in space should be able to communicate instantaneously. We observe this not to be the case. From what we know if two particles have not interacted in the past they should not be able to communicate. Hence the monogamy of entanglement we observe could be interpreted as simply a low-energy approximation. The reason points to the ER=EPR relation. As it has been suggested by Susskind and Maldacena any pair of entangled particles should have a tiny wormhole connecting them. The new view regarding the structure of the spacetime strongly advocates this proposal. An integral feature of the combinatorial structure of the spacetime should be namely the possibility for creating tiny wormholes between any pair of entangled particles.

\section{Acknowledgements}

The author is grateful to Prof. Lachezar Filipov, Head of Astrophysics Department at the Bulgarian Academy of Sciences, for the productive discussions.

\section{References}

[1] Guth, A.H. (1981) Inflationary Universe: A Possible Solution to the Horizon and Flatness Problems. Physical Review 
D, 23, 347. http://dx.doi.org/10.1103/physrevd.23.347

[2] Linde, A. (2007) Inflationary Cosmology. arXiv:0705.0164v2 [hep-th].

[3] Tsujikawa, S. (2003) Introductory Review of Cosmic Inflation. arXiv:hep-ph/0304257v1.

[4] Liddle, A.R. (1999) An Introduction to Cosmological Inflation. arXiv:astro-ph/9901124v1.

[5] Planck Collaboration 2013.

[6] Ijjas, A., Steinhardt, P.J. and Loeb, A. (2013) Inflationary Paradigm in Trouble after Planck 2013. arXiv:1304.2785v2 [astro-ph.CO].

[7] Linde, A.D. (1982) A New Inflationary Universe Scenario: A Possible Solution of the Horizon, Flatness, Homogeneity, Isotropy and Primordial Monopole Problems. Physics Letters B, 108, 389. http://dx.doi.org/10.1016/0370-2693(82)91219-9

[8] Albrecht, A. and Steinhardt, P.J. (1982) Cosmology for Grand Unified Theories with Radiatively Induced Symmetry Breaking. Physical Review Letters, 48, 1220. http://dx.doi.org/10.1103/PhysRevLett.48.1220

[9] Hawking, S.W. (1975) Particle Creation by Black Holes. Physics, 43, 199-220. http://dx.doi.org/10.1007/bf02345020

[10] Parikh, M.K. and Wilczek, F. (2000) Hawking Radiation as Tunneling. Physical Review Letters, 85, 5042. http://dx.doi.org/10.1103/PhysRevLett.85.5042

[11] Parikh, M.K. (2004) A Secret Tunnel through the Horizon. arXiv:hep-th/0405160.

[12] Corda, C. (2014) Precise Model of Hawking Radiation from the Tunnelling Mechanism. arXiv:1411.1213v2 [physics. gen-ph].

[13] Corda, C. (2013) Time-Dependent Schrodinger Equation for Black Hole Evaporation: No Information Loss. arXiv:1 304.1899v5 [gr-qc].

[14] Mavromatos, N.E. (1996) Eluding the No-Hair Conjecture for Black Holes. arXiv:gr-qc/9606008v1.

[15] Chrusciel, P.T., Costa, J.L. and Heusler, M. (2012) Stationary Black Holes: Uniqueness and Beyond. arXiv:1205.6112 [gr-qc].

[16] Frampton, P.H. (2015) Cyclic Entropy: An Alternative to Inflationary Cosmology. arXiv:1501.03054v2 [gr-qc].

[17] Lehners, J.-L. (2010) Ekpyrotic Nongaussianity: A Review. Advances in Astronomy, 2010, Article ID: 903907. http://dx.doi.org/10.1155/2010/903907

[18] Ray, M.W., Ruokokoski, E., Kandel, S., Möttönen, M. and Hall, D.S. (2014) Observation of Dirac Monopoles in a Synthetic Magnetic Field. arXiv:1408.3133v1 [cond-mat.quant-gas].

[19] 'tHooft, G. (1999) Quantum Gravity as a Dissipative Deterministic System. arXiv:gr-qc/9903084v3.

[20] Van Raamsdonk, M. (2010) Building up Spacetime with Quantum Entanglement. arXiv:1005.3035v1 [hep-th].

[21] Maldacena, J. and Susskind, L. (2013) Cool Horizons for Entangled Black Holes. arXiv:1306.0533v2 [hep-th]. 